\title{
Successful Imatinib Therapy for Neuroendocrine Carcinoma With Activating KIT Mutation: A Case Study
}

\author{
James Perkins, $\mathrm{MD}^{\mathrm{a}}$; Patrick Boland, $\mathrm{MD}^{\mathrm{b}}$; Steven J. Cohen, MDª Anthony J. Olszanski, RPh, MD; \\ Yan Zhou, PhDc; Paul Engstrom, MDa; and Igor Astsaturov, MD, PhD ${ }^{\mathrm{a}, \mathrm{d}}$
}

\begin{abstract}
Neuroendocrine tumors (NET) and gastrointestinal stromal tumors (GIST) are believed to originate from the cells of Cajal that are randomly dispersed along the aerodigestive tract. Despite their distinct morphologic appearance, NET and GIST may share oncogenic mechanisms. Often presenting in the metastatic setting, treatment options for patients with NET are limited. This case report presents a patient with refractory metastatic NET that did not respond conventional chemotherapy. The patient was treated with a KIF11 inhibitor in a phase I clinical trial and experienced a prolonged and clinically meaningful partial response. On progression at 20 months, the patient's tumor was sequenced to reveal a KIT exon 11 mutation. Institution of imatinib therapy achieved a rapid and sustained antitumor effect with profound clinical benefit. Despite previously reported KIT expression in NET, this is the first documented case of an activating KIT mutation in NET and of successful treatment with both a KIF11 inhibitor and imatinib, each of which was elucidated through molecular profiling of the patient's tumor. Imatinib may be a valuable therapy in NET harboring activating KIT mutations. (J Natl Compr Canc Netw 2014;12:847-852)
\end{abstract}

\section{NCCN: Continuing Education}

\section{Accreditation Statement}

This activity has been designated to meet the educational needs of physicians and nurses involved in the management of patients with cancer. There is no fee for this article. No commercial support was received for this article. The National Comprehensive Cancer Network (NCCN) is accredited by the ACCME to provide continuing medical education for physicians.

From the aDepartment of Medical Oncology, Fox Chase Cancer Center, Philadelphia, Pennsylvania; ${ }^{b}$ Department of Medicine, Division of Medical Oncology, Roswell Park Cancer Institute, Buffalo, New York; 'Biostatistics and Bioinformatics Facility, Fox Chase Cancer Center, Philadelphia, Pennsylvania; and d Deptartment of Biochemistry, Kazan Federal University, Russia.

Submitted August 6, 2013; accepted for publication November 4, 2013. Dr. Olszanski has disclosed that he is an advisor for Santaris Pharma A/S and Millennium Pharmaceuticals, Inc. Dr. Astsaturov has disclosed that he is a consultant for Caris Life Sciences, Ltd., and received partial support from The Program of Competitive Growth of Kazan Federal University, Russian Federation. The remaining authors have disclosed that they have no financial interests, arrangements, affiliations, or commercial interests with the manufacturers of any products discussed in this article or their competitors. Correspondence: Igor Astsaturov, MD, PhD, Fox Chase Cancer Center, Department of Medical Oncology, 333 Cottman Avenue, Philadelphia, PA 19111. E-mail: igor.astsaturov@fccc.edu
NCCN designates this journal-based CME activity for a maximum of 1.0 AMA PRA Category 1 Credit(s) $^{\mathrm{TM}}$. Physicians should claim only the credit commensurate with the extent of their participation in the activity.

NCCN is accredited as a provider of continuing nursing education by the American Nurses Credentialing Center's Commission on Accreditation.

This activity is accredited for 1.0 contact hours. Accreditation as a provider refers to recognition of educational activities only; accredited status does not imply endorsement by NCCN or ANCC of any commercial products discussed/displayed in conjunction with the educational activity. Kristina M. Gregory, RN, MSN, OCN, is our nurse planner for this educational activity.

All clinicians completing this activity will be issued a certificate of participation. To participate in this journal CE activity: 1) review the learning objectives and author disclosures; 2 ) study the education content; 3 ) take the posttest with a $66 \%$ minimum passing score and complete the evaluation at http://education.nccn.org/ node/46926; and 4) view/print certificate.

Release date: June 16, 2014; Expiration date: June 16, 2015

\section{Learning Objectives}

Upon completion of this activity, participants will be able to:

- Summarize the criteria on which the classification of NET is based and list some markers for NET

- Describe the rationale for the use of imatinib in the treatment of patients with NET with activating KIT mutations

- Evaluate the data supporting the hypothesis that NET and GIST arise from a common precursor

EDITOR

Kerrin M. Green, MA, Assistant Managing Editor, JNCCN_Journal of the National Comprehensive Cancer Network

Ms. Green has disclosed that she has no relevant financial relationships.

\section{CE AUTHORS}

Deborah J. Moonan, RN, BSN, Director, Continuing Education \& Grants Ms. Moonan has disclosed that she has no relevant financial relationships.

Ann Gianola, MA, Manager, Continuing Education \& Grants Ms. Gianola has disclosed that she has no relevant financial relationships. Kristina M. Gregory, RN, MSN, OCN, Vice President, Clinical Information Operations

Ms. Gregory has disclosed that she has no relevant financial relationships. 
Neuroendocrine tumors (NET) and gastrointestinal stromal tumors (GIST) are believed to originate from the cells of $\mathrm{Cajal}^{1-3}$ that are randomly dispersed along the aerodigestive tract. Despite their distinct morphologic appearance, NET and GIST may share oncogenic mechanisms. Often presenting in the metastatic setting, patients with NET have limited treatment options. This case report presents a patient with refractory metastatic NET that did not respond conventional chemotherapy. The patient was treated with a kinesin 11 (KIF11) inhibitor in a phase I clinical trial and experienced a prolonged and clinically meaningful partial response. On progression at 20 months, the patient's tumor was sequenced to reveal a KIT exon 11 mutation. Institution of imatinib therapy achieved a rapid and sustained antitumor effect with profound clinical benefit. Despite previously reported KIT expression in NET, this is the first documented case of an activating KIT mutation in NET and of successful treatment with both a KIF11 inhibitor and imatinib, each of which was elucidated through molecular profiling of the patient's tumor. Imatinib may be a valuable therapy in NET harboring activating KIT mutations.

\section{Case Report}

A 48-year-old man with a past medical history of hypertension and testicular cancer in 1988, which was successfully treated with radiation and surgery, presented in August 2009 to a local emergency department with severe right upper-quadrant pain exacerbated with cough and inspiration without flushing or diarrhea. A CT scan revealed an enlarged liver extending to the pelvis with numerous hypodense metastatic foci without evidence of a primary tumor (Figure 1A). Fine-needle aspiration (FNA) biopsy of the liver demonstrated neuroendocrine carcinoma

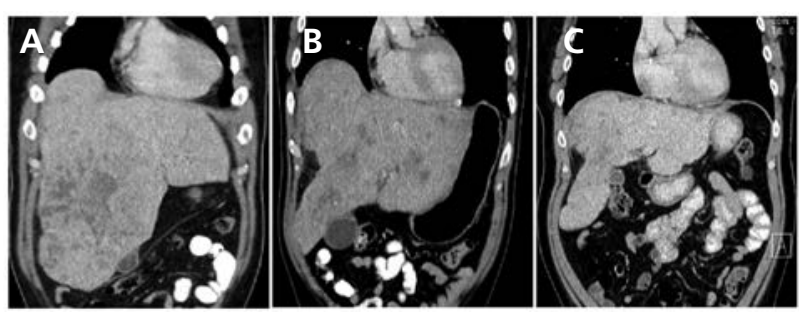

Figure $1 \mathrm{CT}$ images of the abdomen after oral and intravenous contrast administration at baseline (A), after 3 months of KIF11 inhibitor therapy (B), and after 4 months of imatinib therapy (C). with rare mitoses. The amount of biopsy material was insufficient to obtain $\mathrm{Ki}-67$ or any other testing. He underwent initial treatment with 4 cycles of cisplatin and etoposide, which produced minimal to no improvement. Four months later he presented to Fox Chase Cancer Center to review further treatment options. The patient received 8 months of 5 -fluorouracil and streptozocin-based chemotherapy without significant improvement. ${ }^{4}$ His physical examination at that time was remarkable for painful hepatomegaly, with a liver edge palpable below the umbilicus as measured at the mid-sternal line. Laboratory tests revealed an elevated chromogranin A level at 40.0 $\mathrm{ng} / \mathrm{mL}$ (normal range, 1.9-15.0 ng/mL; Figure 2).

In 2010, sunitinib and everolimus were not available for treatment outside of clinical trials. Additionally, the patient had no identifiable pancreatic mass to suggest a pancreatic origin of the extensive metastatic process involving the liver. Therefore, in an effort to identify putative drug targets for this patient, the authors conducted in silico comparisons of global mRNA profiles of NET and colon adenocarcinoma using publicly available Gene Expression Omnibus data sets (GSE2109). This showed enrichment of NET samples for several transcripts known to be bona fide markers of neuroendocrine cells: SV2C (synaptic vesicle glycoprotein 2C), CHGA, CHGB (chromogranin A and B), and TPH1 (tryptophan hydroxylase), supporting the overall validity of the approach. Another family of molecules highly expressed in NET compared with adenocarcinoma (false discovery rate $<20 \%$ ) were kinesin family proteins $1 \mathrm{~A}, 3 \mathrm{~A}$, and $5 \mathrm{C}$. Based on this information, the patient was enrolled in a phase I clinical trial using an inhibitor of KIF11.

Shortly after the initiation of the clinical trial treatment, a rapid improvement in symptoms was documented: pain decreased markedly, obviating the need for narcotics, and the patient's body weight increased back to baseline secondary to improvements in appetite. Consistent with the observed clinical benefit, blood chromogranin A levels precipitously declined (Figure 2). Physical examination confirmed decreased pain and a progressive reduction of the liver vertical size on palpation beginning from week 4 of therapy. On repeat imaging, a partial response was confirmed after 3 months of KIF11 inhibitor therapy by RECIST criteria (Figure 1B). ${ }^{5}$ Overall, the patient gained 20 pounds and his abdominal pain 


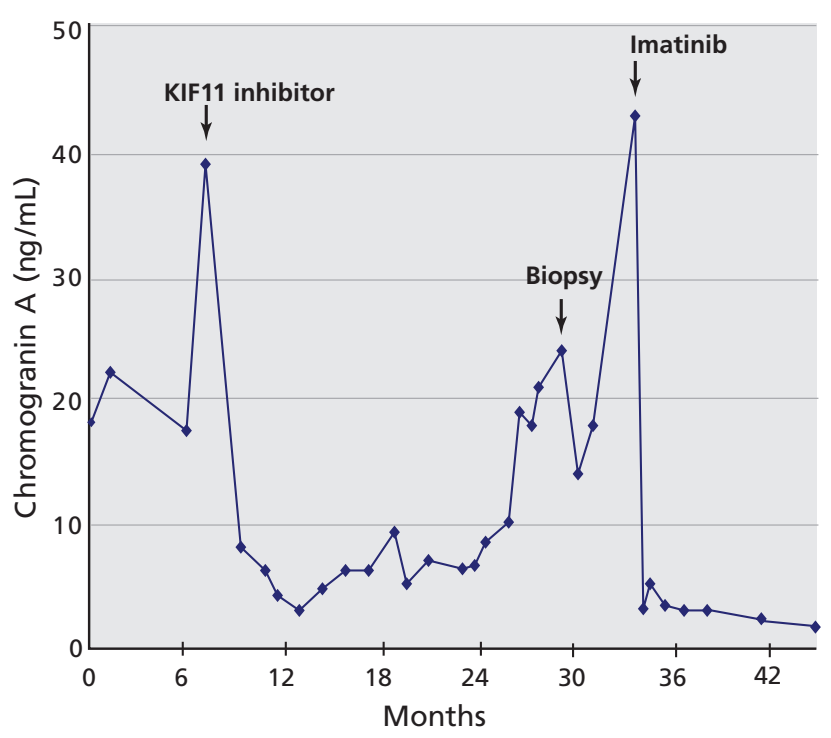

Figure 2 Serum chromogranin A measurements (reference range, $1.9-15.0 \mathrm{ng} / \mathrm{mL}$ ).

subsided to 3 on a scale of 10 . This response lasted for 20 months, followed by slow tumor progression as manifested by recurrence of abdominal pain, rising chromogranin A levels (Figure 2), and more than a $25 \%$ increase in target lesion size.

Because of a shortage of cellular material in the initial FNA, a second core needle biopsy of the liver was obtained in June 2012 and a comprehensive analysis of putative tumor biomarkers (Caris molecular profiling assay, Caris Life Sciences, Inc., Irving, TX) was ordered. The second biopsy revealed an intermediate-grade neuroendocrine carcinoma (Figure 3), staining positive for chromogranin and synaptophysin, with $20 \%$ of tumor cells positive for $\mathrm{Ki}-67$. Targeted sequencing of the tumor DNA revealed an activating mutation in exon 11 (V560del, c.1679_1681delTTG) of the KIT receptor tyrosine kinase. ${ }^{6}$ This mutation has been previously associated with efficacy of imatinib in patients with GIST. ${ }^{7}$ A splice variant of BAP1 $(2056+1 \mathrm{C}>\mathrm{T})$, a gene associated with familial cancer risk, ${ }^{8}$ and amplification of MCL1 were detected in the tumor DNA. Immunohistochemical evaluation of KIT expression in the tumor revealed strongly $(3+)$ positive staining on $100 \%$ of cells (Figure 3), reinforcing the idea that, in this case, KIT overexpression and the presence of an activating mutation represented the central mechanism of this patient's carcinoma. Based on this information, therapy was initiated with imatinib at a standard dose of $400 \mathrm{mg} / \mathrm{d}$. After 2 weeks of imatinib therapy, the patient's pain began to subside and the chromogranin A declined from 44 to $4 \mathrm{ng} / \mathrm{mL}$. On physical examination after 2 months of imatinib, the patient's recurrent hepatomegaly resolved. A CT scan of the liver confirmed a marked reduction of the hepatic lesions and normalization of the organ size (Figure 1A). At the time of writing, 46 months after his initial diagnosis, the patient had been continuing on imatinib at $400 \mathrm{mg} / \mathrm{d}$ for 12 months with good tolerability. Chromogranin A level at 12 months remains low at $4 \mathrm{ng} / \mathrm{mL}$.

\section{Discussion}

The number of reported cases of NET in the United States from 1975 to 2004 is more than 100,000, which is more than that of pancreatic cancer and stomach cancer combined. Since 1973, the incidence of neuroendocrine cancer has increased more than 5-fold. Most NET occur in the gastrointestinal tract, with nearly $42 \%$ occurring in the small bowel. ${ }^{9}$ These tumors are classified based on site of origin and grade, as assessed by mitotic count and Ki-67 score. Welldifferentiated tumors (low- and intermediate-grade, corresponding to a $\mathrm{Ki}-67$ index of $<3 \%$ and $3 \%-20 \%$, respectively) behave and are treated markedly differently from poorly differentiated NET. A recent analysis of SEER data shows the median survival of well-differentiated NETs with distant metastases to be 33 months. This decreases to only 5 months for tumors that are poorly differentiated with distant metastases. Although increasing grade portends a worse prognosis and ultimately dictates a differing treatment approach, great heterogeneity remains even among well-differentiated NET. Finding therapeutic vulnerabilities in NET is of high clinical importance. For example, a subset of NET (mostly of the foregut origin) is characterized by low expression of O-6-methylguanine-DNA methyltransferase (MGMT), which portends greater sensitivity to alkylating agents such as temozolomide and streptozocin. ${ }^{10}$

This patient presented with a large hepatic tumor burden, making aggressive treatment via surgical debulking of the liver a poor option. Not surprisingly, treatment with aggressive chemotherapeutic regimens resulted in modest success consistent with the limited studies of chemotherapy in metastatic carcinoid tumors. ${ }^{11}$ However, the subsequent response and clinical benefit that the patient experienced sec- 


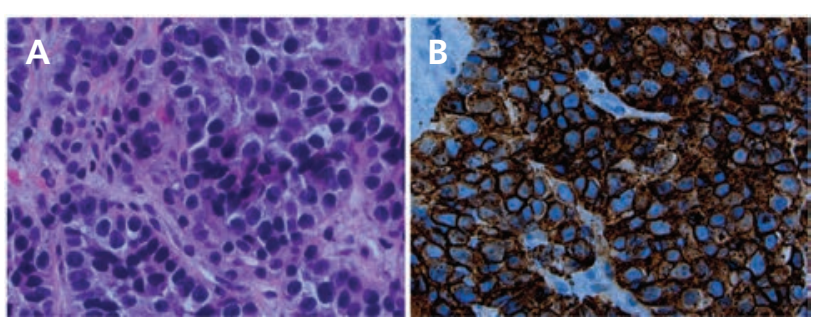

Figure 3 Hematoxylin-eosin (A) and immunohistochemical (B) evaluation of KIT expression in a core biopsy of a hepatic metastasis.

ondary to treatment with the KIF11 inhibitor was both surprising and remarkable.

KIF11 is a homotetrameric protein that attaches to antiparallel microtubules, causing formation of a bipolar spindle allowing complete separation of chromosomes during mitosis. Inhibition of KIF11 results in a monopolar spindle apparatus causing incomplete separation of chromosomes and apoptosis. ${ }^{12}$ The present case is the first documentation in the literature of a kinesin-5 inhibitor providing clinical benefit to a patient with a metastatic NET. Overexpression of KIF11 and its subsequent use as a therapeutic target in bladder cancer has been documented, but this strategy is not considered to be a standard treatment option. ${ }^{13}$ The mechanism through which KIF11 inhibition produced such a dramatic response in the present patient, whose tumor was refractory to chemotherapy, remains to be elucidated.

The NET in this patient also displayed high expression of KIT via immunohistochemistry. Expres- sion of KIT has been variable, as shown in the few preclinical studies performed of NET. KIT expression by immunohistochemistry in carcinoid tumors ranges from $0 \%$ to $38 \% .{ }^{14,15}$ Based on these data and the high expression of platelet-derived growth factor receptors (PDGFR) $\alpha$ and $\beta$ in carcinoid tumors, a phase II trial of imatinib in an unselected population of patients with these tumors was previously conducted. Imatinib is a small molecule inhibitor of multiple tyrosine $\mathrm{ki}$ nases, including KIT, PDGFR, and ABL. In this trial, a partial response was seen in just 1 patient (4\%). Although this response was durable, it was not associated with KIT overexpression on immunohistochemistry analysis. However, an additional $63 \%$ of patients in this trial experienced stable disease, with a median progression-free survival (PFS) of 5.9 months, and 7 patients $(26 \%)$ experienced a biochemical response, resulting in a significantly improved PFS compared with those without a biochemical response (115 vs 24 weeks; $P=.003) .{ }^{14}$ In an unselected population, some signal of efficacy was thus seen, but this treatment confers only limited benefit to this patient cohort as a whole. Imatinib has not been further pursued in this disease. Furthermore, the present patient's tumor was found to have a "classical" exon 11-activating mutation of KIT (Figure 4). Notably, KIT mutations are seen rarely, but randomly, in a variety of human cancers other than GIST in which the therapeutic significance of these mutations remains unclear. In the
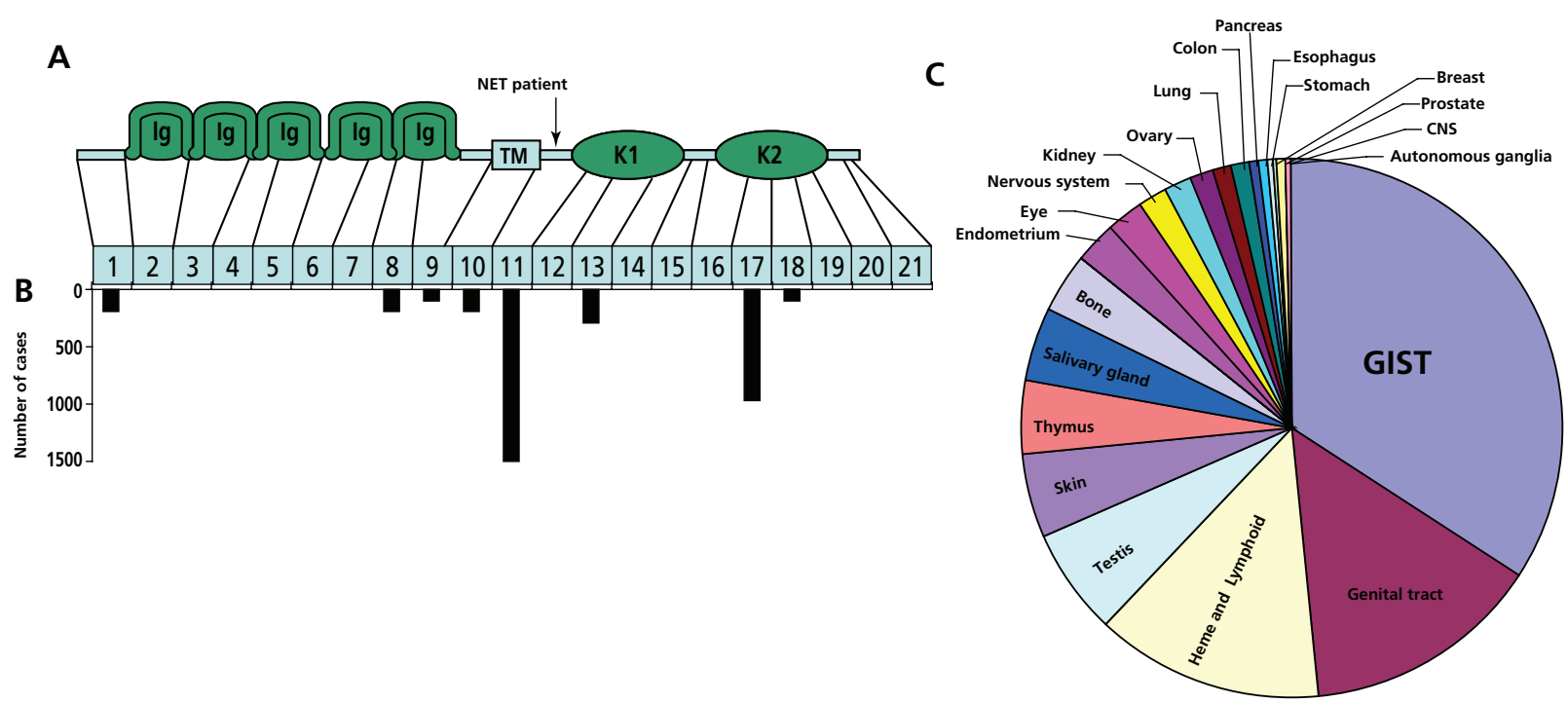

Figure 4 (A) Schema of KIT protein domains (green) and gene exons (light blue) and (B) the prevalence of mutations in KIT relative to exons. (C) Frequency of KIT gene mutations in different tumor types.

Abbreviations: CNS, central nervous system; GIST, gastrointestinal stromal tumors; NET, neuroendocrine tumors.

(B, C) Data from the COSMIC database. Available at: http://cancer.sanger.ac.uk/cancergenome/projects/cosmic/. Accessed May 5, 2014. 
present patient, the use of imatinib led to impressive symptomatic and radiographic improvements during his current 9-month treatment.

The interstitial cells of Cajal, the "pacemaker cells" of the gastrointestinal tract, likely give rise to GIST. ${ }^{16}$ Huizinga et $\mathrm{a}^{17}$ showed that knockout of KIT in a mouse model results in loss of cells of Cajal. Three years after this discovery, Hirota et $\mathrm{al}^{18}$ published their findings of KIT mutations in GIST. Interestingly, case reports have described the simultaneous occurrence of mixed GIST and neuroendocrine carcinomas in the same tumor, suggesting the existence of a shared precursor giving rise to both cancers. ${ }^{3,19,20}$ A subset of GIST arises from autonomous ganglia (gastrointestinal autonomic nerve tumors [GANT] ${ }^{21}$ ) of proximal bowel (stomach, small intestine, and mesentery). Some GANT and GIST may present with epithelioid microscopic features, but nearly uniformly demonstrate gastrointestinal tract involvement and are almost uniformly synaptophysin and chromogranin-negative, ${ }^{22}$ in contrast to the presence of both neuroendocrine biomarkers expressed in the present case. This may give merit to the idea that GIST and NET tumors divert from a common precursor, and in so doing, may prompt clinicians to search for KIT mutations in NET.

\section{Conclusions}

This report describes the treatment benefit afforded to a patient with a metastatic NET carcinoma through the use of 2 novel therapeutic agents. Available mRNA profiling data demonstrated a rationale for inhibition of kinesin family proteins in carcinoid tumors. A phase I trial involving the use of an inhibitor of kinesin-5 produced clinically meaningful symptomatic improvement and a partial response with a 20-month durability. Subsequently, at the time of progression, molecular analysis revealed an unexpected finding: KIT overexpression associated with a KIT exon 11 mutation. The present patient is currently continuing on treatment with imatinib, with evidence of a second clinical benefit and a corresponding biochemical response. Although the durability of his benefit remains to be seen, the authors feel that these data further support efforts to pursue detailed molecular characterization of individual tumors in efforts to improve cancer treatment. This may serve not only to identify new therapeutic tar- gets but also to tease out the subset of patients who truly derive benefit from largely discarded therapeutic avenues.

\section{References}

1. Bümming $P, N$ ilsson $O, A$ hlman $H$, et al. Gastrointestinal stromal tumors regularly express synaptic vesicle proteins: evidence of a neuroendocrine phenotype. Endocr Relat Cancer 2007;14:853863.

2. Grande E, Capdevila J, Barriuso J, et al. Gastroenteropancreatic neuroendocrine tumor cancer stem cells: do they exist? Cancer Metastasis Rev 2012;31:47-53.

3. Samaras VD, Foukas PG, Triantafyllou K, et al. Synchronous well differentiated neuroendocrine tumour and gastrointestinal stromal tumour of the stomach: a case report. BMC Gastroenterol 2011;11:27.

4. Engstrom PF, Lavin PT, Moertel CG, et al. Streptozocin plus fluorouracil versus doxorubicin therapy for metastatic carcinoid tumor. J Clin Oncol 1984;2:1255-1259.

5. Therasse P, Arbuck SG, Eisenhauer EA, et al. New guidelines to evaluate the response to treatment in solid tumors. European Organization for Research and Treatment of Cancer, National Cancer Institute of the United States, National Cancer Institute of Canada. J Natl Cancer Inst 2000;92:205-216.

6. Ashfaq R. Molecular profiling for personalized cancer care. Clin Exp Metastasis 2012;29:653-655.

7. Heinrich MC, Griffith DJ, Druker BJ, et al. Inhibition of c-kit receptor tyrosine kinase activity by STI 571 , a selective tyrosine kinase inhibitor. Blood 2000;96:925-932.

8. Wadt K, Choi J, Chung JY, et al. A cryptic BAP1 splice mutation in a family with uveal and cutaneous melanoma, and paraganglioma. Pigment Cell Melanoma Res 2012;25:815-818.

9. Yao JC, Hassan M, Phan A, et al. One hundred years after "carcinoid": epidemiology of and prognostic factors for neuroendocrine tumors in 35,825 cases in the United States. J Clin Oncol 2008;26:3063-3072.

10. Kulke MH, Hornick JL, Frauenhoffer C, et al. O6-methylguanine DNA methyltransferase deficiency and response to temozolomidebased therapy in patients with neuroendocrine tumors. Clin Cancer Res 2009;15:338-345.

11. Mitry E, Baudin E, Ducreux M, et al. Treatment of poorly differentiated neuroendocrine tumours with etoposide and cisplatin. Br J Cancer 1999;81:1351-1355.

12. Sarli V, Giannis A. Targeting the kinesin spindle protein: basic principles and clinical implications. Clin Cancer Res 2008;14:7583-7587.

13. Ding S, Nishizawa K, Kobayashi $T$, et al. A potent chemotherapeutic strategy for bladder cancer: (S)-methoxytrityl-L-cystein, a novel Eg5 inhibitor. J Urol 2010;184:11751181.

14. Yao JC, Zhang JX, Rashid A, et al. Clinical and in vitro studies of imatinib in advanced carcinoid tumors. Clin Cancer Res 2007;13:234-240.

15. Lankat-Buttgereit B, Horsch D, Barth P, et al. Effects of the tyrosine kinase inhibitor imatinib on neuroendocrine tumor cell growth. Digestion 2005;71:131-140. 
16. Min KW, Leabu M. Interstitial cells of Cajal (ICC) and gastrointestinal stromal tumor (GIST): facts, speculations, and myths. J Cell Mol Med 2006;10:995-1013.

17. Huizinga JD, Thuneberg L, Kluppel M, et al. W/kit gene required for interstitial cells of Cajal and for intestinal pacemaker activity. Nature 1995;373:347-349.

18. Hirota S, Isozaki K, Moriyama Y, et al. Gain-of-function mutations of c-kit in human gastrointestinal stromal tumors. Science 1998;279:577-580.

19. Lin YL, Wei CK, Chiang JK, et al. Concomitant gastric carcinoid and gastrointestinal stromal tumors: a case report. World J Gastroenterol 2008;14:6100-6103.
20. Maiorana A, Fante R, Maria Cesinaro A, et al. Synchronous occurrence of epithelial and stromal tumors in the stomach: a report of 6 cases. Arch Pathol Lab Med 2000;124:682-686.

21. Lauwers GY, Erlandson RA, Casper ES, et al. Gastrointestinal autonomic nerve tumors. A clinicopathological, immunohistochemical, and ultrastructural study of 12 cases. Am J Surg Pathol 1993;17:887-897.

22. Miettinen M, Makhlouf $\mathrm{H}$, Sobin $\mathrm{LH}$, et al. Gastrointestinal stromal tumors of the jejunum and ileum: a clinicopathologic, immunohistochemical, and molecular genetic study of 906 cases before imatinib with long-term follow-up. Am J Surg Pathol 2006;30:477-489.

\section{Instructions for Completion}

To participate in this journal CE activity: 1) review the learning objectives and author disclosures; 2 ) study the education content; 3 ) take the posttest with a $66 \%$ minimum passing score and complete the evaluation at http://education.nccn.org/ node/46926; and 4) view/print certificate. After reading the article, you should be able to answer the following multiple- choice questions. Credit cannot be obtained for tests completed on paper. You must be a registered user on NCCN.org. If you are not registered on NCCN.org, click on "New Member? Sign up here" link on the left hand side of the Web site to register. Only one answer is correct for each question. Once you successfully answer all posttest questions you will be able to view and/or print your certificate. Software requirements: Internet.

\section{Posttest Questions}

1. NET are classified by which of the following?
a. $\mathrm{Ki}-67$
b. Chromogranin level
c. Kinesin level
d. Tumor cell anaplasia

2. Patients with metastatic poorly differentiated NET of the gastrointestinal tract are typically considered for initial treatment via a platinum doublet (carboplatin or cisplatin plus etoposide), with high-grade pathology determined by the presence of a mitotic rate of $>20$ mitoses per high-pow- ered field or a Ki-67 greater than:
a. $10 \%$
b. $15 \%$
c. $20 \%$

3. Which of the following drugs is NOT used to treat cancer positive for KITactivating mutations:

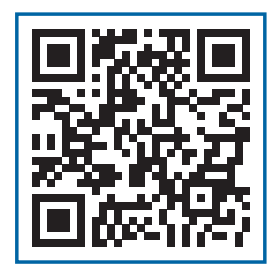
a. Everolimus
b. Sunitinib
c. Imatinib
d. Dasatinib 